Global Journal of Pure and Applied Mathematics.

ISSN 0973-1768 Volume 16, Number 2 (2020), pp. 305-324

(C) Research India Publications

https://dx.doi.org/10.37622/GJPAM/16.2.2020.305-324

\title{
Applications of Soft Set in Decision Making Problems
}

\author{
B.V.S.T.Sai \\ Research Scholar \\ Department of Mathematics, A.U. College of Science and Technology \\ Andhra University, Visakhapatnam-530003, A.P. State, India.
}

\begin{abstract}
In this paper we present some of the main developments in the Applications of soft set theory as a survey of literature motivated by Molodtsov.

Keywords: Fuzzy set, Soft set, Bijective soft set, Bipolar soft sets, Hesitant fuzzy soft set ,Interval soft set,npn-soft sets,probabilistic soft sets, Intuitionistic fuzzy soft sets, Vague soft set, hesitant soft set, N-soft set, Hesitant fuzzy Nsoft sets and Incomplete fuzzy soft set.
\end{abstract}

\section{INTRODUCTION}

In 1965 L. A. Zadeh [1] coined his remarkable theory of Fuzzy sets that deals with a kind of uncertainty known as "Fuzziness" and which is due to partial membership of an element in a set. Although Fuzzy set theory is very successful in handling uncertainties arising from vagueness or partial belongingness of an element in a set, it cannot model all sorts of uncertainties prevailing in different real physical problems. Thus search for new theories has been continued. In 1982 Z. Pawlak[2] came up with his Rough set theory. In 1999, Molodtsov [3]initiated a novel concept of soft set theory, which is a completely new approach for modeling vagueness and uncertainty. Molodtsov successfully applied the soft set theory into several directions such as smoothness of functions, game theory, Riemann integration, Perron integration, theory of measurement and so on.

A soft set is a classification of elements of the universe with respect to some given set of parameters. It has been shown that soft set is more general in nature and has more 
capabilities in handling uncertain information. Also a fuzzy set or a rough set can be considered as a special case of soft sets. Research involving soft sets and its application in various fields of science and technology are currently going on in a rapid pace.

Maji-Roy [4] gave first practical application of soft sets in decision making problems and defined soft binary operations like AND, OR, Union, Intersection of two soft sets. Xiao-Chen [5] gave an appropriate definition and a method was designed for recognizing soft information patterns by establishing the information table based on soft sets theory. Mappings on soft sets are defined by Kharal-Ahmad [6]. CagmanEnginoglu [7] redefined the operations of Molodstov's soft sets to make them more functional for improving several new results. They also constructed uni-int decision making method for the AND product by using uni-int decision making function. AktasCagman [8] defined soft sets and soft groups. In 2009 Ali-Feng [9] gave some operations in the soft sets. Further, Cagman-Enginoglu [10] defined soft matrices and their operations. Consequently they established min - max decision making methods for OR, AND, AND-NOT and OR-NOT product operators of the soft sets and discussed the applications with suitable examples in the field of Agriculture, Medicine and Social science.Chen[11] proposed parameterization reduction of soft sets and then Kong [12]presented the normal parameterization reduction of soft sets. Xiao [13] studied synthetically evaluating method for business competitive capacity and gave recognition for soft information based on the theory of soft sets. Zou-Xiao [14] presented data analysis approaches of soft sets under incomplete information. Pei-Miao [15] showed that the soft sets are a class of special information systems.

Mushrif [16] presented a new algorithm based on the notions of soft set theory for classification of the natural textures. Kovkov [17] considered the optimization problems in the framework of the theory of soft sets which is directed to formalization of the concept of approximate object description. Majumdar-Samanta [18] studied the similarity measure of soft sets. Maji [19] presented the concept of the fuzzy soft sets (fs-sets) by embedding the ideas of fuzzy sets. By using this definition of fs-sets many interesting applications of soft set theory have been expanded. Bhardwaj [20] used reduct soft set for real life decision making problems. Yang-Xiao [21] defined the operations on fuzzy soft sets which are based on three fuzzy logic operators: negation, triangular norm and triangular conorm.

Crossingham-Marwala [22] presented an approach to optimize rough sets partition sizes using four optimization techniques namely genetic algorithm, particle swarm optimization, hill climbing and simulated annealing for interstate conflict.

Jiang -Tang-Chen [23] proposed an extension of soft set theory by using the concepts of Description Logics (DLs) to act as the parameters of soft set and Jiang proposed an adjustable approach to intuitionistic fuzzy soft sets based on decision making. Das and Kar [24] proposed an algorithm approach based on intuitionistic fuzzy soft set (IFSS) in the group decision making (GDM) which explores a particular disease reflecting the agreement of all experts. Agarwal-Garg [25] extended the Intuitionistic Fuzzy Soft Sets (IFSS) to generalized IFSS (GIFSS) by introducing the generalization parameter to the 
pool of the intuitionistic fuzzy numbers (IFNs) of IFSS and demonstrated in decision making area.

Feng-Jun [26] proposed an adjustable approach to fuzzy soft set based decision making, and discussed the application of interval-valued fuzzy soft sets in decision making problems. Feng-Zhou [27] introduced soft set discernibility in soft sets to solve the problems of decision making. Deli - Broumi [28] presented neutroshopic soft sets for decision making called NSM-decision making, while Deli combined an interval-valued neutrosophic sets and a soft sets called $i v n$-soft sets and implemented it on decision making problems.

Maji-Roy [29] proposed fuzzy soft sets and presented an application of fuzzy soft set theory in decision making problem. Furthermore, Alcantud [30] proposed a novel approach of fuzzy soft set in decision making in the presence of multi observer input parameter data sets. Majumdar - Samanta [31] introduced the concept of generalized fuzzy soft sets. Xiao-Yang [32] proposed the concept of D-S generalized fuzzy soft set by combining Dempster-Shafer theory of evidence and generalized fuzzy soft sets.

Deng-Wang [33] proposed an object-parameter approach in incomplete fuzzy soft sets for predicting unknown data. Cagman - Deli [34] defined $t$-norm and $t$-conorm of fuzzy parameterized soft sets (FP-soft sets) and investigated their properties. Cagman-Deli [35] defined means of FP-soft sets and constructed FP-soft sets on decision making methods. Deli- Cagman [36] constructed intuitionistic fuzzy parametrized soft sets (intuitionistic FP-soft sets) for decision making. Deli -Cagman [37] proposed fuzzy soft games and applied it to financial problems.

Gong-Wang [38] proposed bijective soft set under fuzzy environment for decision system based parameters reduction. Wang - Qu [39] introduced axiomatic definitions of entropy, similarity measure and distance measure of vague soft sets. Rosenfeld [40] introduced the concept of fuzzy graph theory. Akram - Nawaz [41] introduced fuzzy soft graphs and investigated the application in employee selection for an organisation.

Mao-Yao-Wang[42] presented the concept of intuitionistic fuzzy soft matrix(IFSM) and applied it in group decision making problem. Yang-Chenli[43] initiated a matrix representation of a fuzzy soft set and applied it in certain decision making problems. The concept of fuzzy soft matrix theory was studied by Borah-Neog [44] and Rajarajeswari-Dhanalakshmi [45] defined intuitionistic fuzzy soft matrix.

\section{A Brief Review on Applications of Soft Sets}

In 2003 Chen-China-Tsang-Yeung [11] proposed a reasonable definition of parameterization reduction of soft sets and compared it with the concept of attributes reduction in rough sets theory. By using this new definition of parameterization reduction, they improved the application of a soft set in a decision making problem. 
Parameterization reduction is also possible in soft sets and related applications as presented by Chen and they further said that the approach followed by Maji was incorrect and also claimed that the reduct is not same for soft set theory and rough set theory. Their idea for reduction of attributes in soft sets was based on the optimal choice concept that addresses the problems of sub-optimal solutions. This problem was also analyzed by Kong-Gao-Wang-Li [47] in 2008 and defined actual parameter reduction that can overcome the problems of sub-optimality.

In 2005 Pei - Miao [15] explored information systems and soft sets in terms of relationship between them. The results of their experiments reveal that information systems and partition-type soft sets share a common formal structure. For instance fuzzy information systems and fuzzy soft sets are equal.

In 2007 Roy-Maji[48] presented a novel method of object recognition from an imprecise multiobserver data. This method involves construction of a Comparison Table from a fuzzy soft set in a parametric sense for decision making.

For decision making using soft set where there is data deficiency Zou- Xiao [49] proposed a novel technique in 2008 and this technique is based on computation of weighted average as per the distribution objects.

In 2009 Herawan- Mustafa[50 ] proved that every rough set can be utilized as soft set for mining purposes. For this reason an alternative approach was proposed by Pawlak to achieve reduct whose results again proved to be same as that of rough reduction

In 2010 Herawan- Rozaida- Deris[51] presented an approach to reduce dimensionality of soft set. The existing solutions on soft set are Boolean- based. However, they may also have non-Boolean values. In case of multi-valued information systems, they presented an alternative approach for reducing attributes. They introduced the idea of multi soft sets that are constructed from multi-valued information systems. Then they also used OR and AND operators on soft sets. They came to know from the experiments that the set of attributes (reduct) required in soft set theory are also same as that of rough set theory.

In 2011 Neog - Sut[52] established new notion of complement of a fuzzy soft set to obtain the solution of a decision problem in an imprecise environment also they applied the notion fuzzy soft matrices and complement of fuzzy soft sets in a decision making problem. Cagman-Enginoglu[53] defined fuzzy soft aggregation operator that allows constructing more efficient decision making method. Finally, they gave an example which shows that the method can be successfully applied to many problems that contain uncertainties.Razak-Mohamad [54] proposed a group decision making method with criteria based on soft set based data mining approach. The weight of each criterion is computed using a method known as Analytic Hierarchy Process (AHP)[55]. The group decision making problem is solved using soft max - min decision making method.Chetia-Das [56] extended Biswas's method for evaluation of answer scripts of students. They assumed five satisfaction levels in order to evaluate the performance of 
students. They include unsatisfactory, satisfactory,good, very good and excellent. They have developed an algorithm that takes student's statistics as input and build a soft set matrix before evaluating the performance of students. Xun Ge -Songlin Yang [57] investigated operations on soft set. They explored the operations defined in the prior works. The results of their work help others to choose right operators and operational rules while working with soft sets. Similarity measure has extensive application in pattern recognition, region extraction,coding theory,image processing and in many other areas. Similarity of two fuzzy soft has been studied by Majumder - Samantha[58]. Sut applied similarity of fuzzy soft sets in decision making problem and used the notion of similarity of fuzzy soft sets in medical diagnosis. Ahmad- Rose-Hassan-Deris[59] proposed two techniques to compare incomplete datasets. The techniques are based on aggregate and calculated support values and parity bits of supported set. When a dataset is downloaded or taken from a source, it might be an incomplete dataset due to VIRUS attacks or any software or hardware problems. As the processing of incomplete datasets will yield inconsistent results, it is essential to know whether the data sets are complete or incomplete prior to using them in data mining algorithms. The results of comparison help in finding missing attributes and take necessary steps to rectify the problems before actually processing the data.

In 2012 Rajpoot- Shailendra -Shrivastava-Abhishek[60] proposed an association rule mining based on soft set approach using constraints with respect to initial support. The constraint is meant for filtering rarely occurred items and false frequent items. As the pruning reduces search space, the dataset is improved and it consumes fewer resources to mine association rules. Afterwards, the dataset is converted to Boolean - valued information system. The resultant dataset is known as soft set. They compared both CSS approach and previous soft set based approach. The results revealed that constraint based soft set approach has exhibited higher performance. Satya Ranjan-Satchidananda [61] applied soft data mining to Bioinformatics and explored the challenges encountered. Of late there has been considerable research on soft sets and their application to Bioinformatics. Soft set theory is used for data analysis and also decision making in Bioinformatics domain. Using adequate parameters membership is decided in soft set theory. Equivalent classes concept is used by rough set theory while the grade of membership is used by fuzzy set theory. Though these three are unique in their functionality, they all commonly deal with vagueness. Thus an idea came up to combine them. The relationship among them was explored by Gorzalzany [62] in 1987. All those techniques are widely used in applications but they have their own limitations as described by Molodtsov .As specified above application of data mining bioinformatics revealed the difficulties such as vagueness, local optimality and intractability. The solution to overcome these drawbacks is to combine soft data mining techniques and apply to bioinformatics in order to achieve best results. Rajarajeswari-Dhanalakshmi [63]applied the notion of similarity between two fuzzy soft sets based on distance, to obtain the solution of a medical problem in an imprecise environment.Bashir-RazakAlkhazaleh[64] introduced the concept of possibility intuitionistic fuzzy soft set and studied some of its properties. Applications of this theory have been given to solve a decision-making problem. Similarity measure of two possibility intuitionistic fuzzy soft 
sets is discussed and an application of this to medical diagnosis has been discussed. The decision making problems with imprecise data has a special significance in real life problems. Das- Borgohain[65] explained the concept of fuzzy soft sets which always possess parameterization tools is applied to solve a multi-observer multi-criteria decision making problem.

Xu-Wang-Hao[66] have developed a theoretical study of the 'Vague Soft Set' (VSS).The combination of Vague Set and Soft Set will be more useful in the field of applications wherever uncertainty appear. Biswas pointed out that one of the chief aim of educational institutions is to provide students with the evaluation reports regarding their test/examination as sufficient as possible and with the unavoidable error as small as possible and presented a fuzzy evaluation method(fem) for applying fuzzy sets in students' answer scripts evaluation. He also modified the fuzzy evaluation method to propose a generalized fuzzy evaluation method (gfem) for students' answer scripts evaluation. Alhazaymeh- Hassan [67]presented the basic properties of vague soft sets and introduced the concept of interval-valued vague soft sets which are an extension of the soft set and its operations such as equality, subset, intersection, union, AND operation, OR operation, complement, and null while further studying some properties and some examples for these concepts. They established number of applications on interval-valued vague soft sets. Lashari-brahim-Senan[68] applied soft set theory to classify sounds of musical instruments. They have done experiments and found the viability of soft set theory for this purpose. Their results revealed that soft set theory can be successfully used for the classification of musical instruments. Thus the soft set theory has got importance in decision making applications. They studied the impact of audio length and frame size on their classification system. Jothi-Inbarani [69] proposed an unsupervised feature selection algorithm using soft set. By using soft set theory the reduction of attributes is achieved. Various datasets are used to test the efficiency of the algorithm in terms of its speed and performance. The experiments were made on the datasets collected from UCI machine learning repository by Blake-Merz[70].

In 2013 Udhaya kumar-Inbarani-Senthil kumar [71] proposed a new approach for generating classification rules using a variant of soft set known as bijective soft sets. The algorithm takes dataset as input and generates a set of rules that help in decision making. Soft set theory has been applied to medical data also. To diagnose the prostate cancer risk. Saziye-Gulnur [72]developed an application of soft sets. In recent years the artificial intelligence has been developed rapidly since it can be applied easily to several areas like medical diagnosis, engineering and economics, among others. In this study they devised a soft expert system (SES) as a prediction system for prostate cancer by using the prostate specific antigen (PSA), prostate volume (PV) and age factors of patients based on fuzzy sets and soft sets and have calculated the patients' prostate cancer risk. Data set has been provided by the Department of Urology, Meram Medical Faculty in Necmettin Erbakan University, Konya, Turkey. Shabir-Naz[73] proposed the notion of bipolar soft sets. Later in 2015 Karaaslan-Karataş[74] redefined the concept of bipolar soft set and bipolar soft set operations as more functional than Shabir and Naz's definition and operations. Also they studied on their basic properties and 
presented a decision making method with application. Majumdar[75 ] introduced the idea of entropy for soft sets and some applications of hybrid soft sets in solving real life problems like medical diagnosis, decision-making, etc. are shown. Issues like measurement of similarity of soft sets are also addressed.

In 2014 Hakim- Sari-Herawan [76] used soft set theory as a generic mathematical tool to describe the objects in the form of information systems and evaluated using multidimensional scaling techniques to find the soft solution and recommendation for making a decision. To obtain the best feasible solution to the problems Gogoi-DuttaChandra [77] considered various parameters relating to the solution .For this they used the best mathematical tool called Fuzzy soft set theory and successfully applied the same in decision making. Wang-Li-Chen[78]extended the classical soft sets to hesitant fuzzy soft sets which are combined by the soft sets and hesitant fuzzy sets. Then, the complement, "AND", "OR", union and intersection operations are defined on hesitant fuzzy soft sets. The basic properties such as DeMorgan's laws and the relevant laws of hesitant fuzzy soft sets are proved. Finally, with the help of level soft set, the hesitant fuzzy soft sets are applied to a decision making problem and the effectiveness is proved by a numerical example. Karaaslan [79] studied neutrosophic soft sets established by Maji and then he redefined notion of neutrosophic soft set and neutrosophic soft set operations. Finally, he presented two applications of neutrosophic soft sets in decision making problem.As a combination of interval set and soft set, Zhang[80] introduced the new notion of interval soft sets and made research on interval soft sets application. He investigated the tabular representation of interval soft sets, introduced the new concepts of interval choice values and applied the theory of interval soft sets to solve a decision making problem by using two methods and discussed the application of soft equality relations in preconditioning of decision making.

In 2015 Irfan Deli[81] firstly defined neutrosophic parameterized neutrosophic soft sets(npn-soft sets) which is combination of a neutrosophic sets and a soft sets. Our npnsoft sets generalizes the concept of the other soft sets such as; fuzzy soft sets, intuitionistic fuzzy soft sets, neutrosophic soft sets, fuzzy parameterized soft sets, intuitionistic fuzzy parameterized soft sets, neutrosophic parameterized soft sets and so on. Then, he introduced some definitions and operations on npn-soft sets and some properties of the sets which are connected to operations have been established. Also, he introduced the concept of npn-soft matrix and their operators which are more functional to make theoretical studies in the npn-soft set theory. Finally, he proposed the decision making method on the npn-soft set theory which can be applied to problems of many fields that contain uncertainty and provided an example that this method can be successfully worked.

Selecting the most appropriate supplier is a key issue in supply chain management and is linked to the success of the entire supply chain. Supplier selection is a multiplecriteria decision-making problem that has qualitative and quantitative factors. The traditional method by which this is calculated adopts a precise value or single linguistic terms to represent attribute data. However, real-life situations have many uncertainties 
and imprecise or missing data with regard to supplier selection. Moreover, experts equivocate between several values in assessing attribute data in real-world situations. These factors increase the difficulty selecting suppliers, causing decision-makers to make incorrect choices. To solve this issue in 2014 Kuei-Hu Chang[82] presented an integrated approach, using a soft set and hesitant fuzzy linguistic term set, for selecting the appropriate supplier in the supply chain. A practical example of liquid crystal display module supplier selection is presented to illustrate the proposed approach, the results of which are compared with those of the arithmetic average method and hesitant fuzzy linguistic term set method. The proposed approach effectively solves the problems of incomplete attribute data and expert hesitation in assessing attribute data.

Congliang-Chunge-xiaolan [83] explained the decision method of basic fuzzy soft set in the application of the asphalt pavement maintenance sorting. In order to determine the order of pavement maintenance plan, according to the survey data of pavement condition: road surface roughness, deflection, pavement damage condition and traffic volume, combined with related soft sets theory and put forward a new decision making method about determination of pavement maintenance scheduling by fuzzy soft set. At last, using this method, combining with example to sort project that need maintenance, the same sort results with other methods are obtained, and the feasibility, ease and simplicity of this method are proved.

Alkhazaleh [84] introduced the concept of multi-interval-valued fuzzy soft set (MIVFSS) and also defined its basic operations, namely complement, union, intersection, AND and OR. Finally, he gave an application of this concept in decision-making problem.

Deli-Cagman [ 85] proposed a game model for dealing with uncertainties which is free of the difficulties. The proposed new game is called a soft game since it is based on soft sets theory. To construct a soft set we can use any parametrization with the help of words and sentences, real numbers, functions, mappings and so on. Therefore, payoff functions of the soft game are set valued function and solution of the soft games obtained by using the operations of sets that make this game very convenient and easily applicable in practice. Deli-Cagman defined two person soft games which can apply to problems contain vagueness and uncertainty. Then they gave four solution methods of the games which are soft saddle points, soft lower and soft upper values, soft dominated strategies and soft Nash equilibrium, also gave an example from the real world. Finally, they extended the two person soft games to n-person soft games.

Sai-Nagaraju [86] proposed framework that deals with uncertainty problems and extracts business intelligence which helps in making well informed decisions. The framework is based on soft sets and can be used in decision support systems of real world. They proposed an algorithm which is part of the underlying framework. This analytical study reveals that the proposed framework is useful to solve real world uncertainty problems. Nase-Nashar [87] presented an application of soft sets in a decision making problem for textile marketing with the help of rough mathematics of Pawlak. Earlier, a rough set representation, and hence, application done by Lin and Yao. 
They used almost analogous representation of the soft sets in the form of a binary information table. Dauda-Mamat- Waziri [88 ] used the concept of AND operation to sort out two best candidates out of five applicants in an interview conducted by a certain bank. Also the identification of Idempotent Property of "AND" and "OR" operation of soft sets is given and proved.

In 2016 Zakri-Hossen-Sharif [89] proposed an Application of Soft sets to diagnose the educational obstacles for students. Cigdem-Hande [90] studied the theory of probabilistic soft sets introduced by Zhu-Wen. They defined equality of two probabilistic soft sets, subset,complement of a probabilistic soft set with examples and also introduced the operations of union, intersection, difference and symmetric difference and proved certain De Morgan's laws hold in probabilistic soft set theory with respect to these new definitions. Garg-Agarwal-Tripathi [25]defined the notion of the fuzzy number intuitionistic fuzzy soft sets, different operations such as union, intersection, complement, max, min, AND and OR have been introduced on fuzzy number intuitionistic fuzzy soft sets environment. Some examples of these operations are given and a few properties are also studied. Mukherjee-Sarkar-Debnath [92] defined intuitionistic fuzzy soft aggregation operator for intuitionistic fuzzy soft set and constructed an algorithm using intuitionistic fuzzy soft aggregation operator for the decision making problem. Finally they applied the algorithm to solve a group decision making problem. Rajesh Kumar Pal [93] aimed to give an alternative way for computation of fuzzy soft decision making problem in more precisely qualitative than the existed methods. By using fs-aggregation method, he obtained the optimum logical results in an easier and faster way. To develop the theory, he first defined fs-sets and their operations then presented the decision making method for the fs-set theory.

Hussain-Irfan-Khan [91] studied the soft set representation of graphs and discussed the application of soft sets to study cycles, circuits, cut vertices, bridges and Hamilton cycles in a graph, some basic definitions regarding multi-set theory and soft set theory are mentioned. Moreover notions about soft set representation of a graph and presented some fundamental concepts of graph theory with the help of soft sets, especially cycles, circuits, cut vertex and bridges. Also they proved that soft sets can be very useful to determine the presence or absence of Hamilton cycles in a given graph.

Hamid-Herawan-Mungad [94] proposed a novel approach for handling conflict situation involving uncertainty. It is based on multi-soft sets taking into account of cooccurrence of parameter related to object in universe. The novelty of the proposed approach is that, unlike in rough set theory that uses decision rules, it is based on the concept of co-occurrence of parameters in soft set theory. They presented an illustrative example on how to handle conflict using multi soft sets. Furthermore, they elaborated the proposed approach of real world dataset of voting from political election data set from Indonesian parliament. However, they achieved lower computational time as compared to rough set approaches.

Nayak-Rath-Mohapatra [95] described that Decision -making is a process to select best out of different alternative possibilities. Every process of decisions produces a final 
choice that may or may not prompt action. It is the study of identifying the best choice according to decision maker. They defined soft relation and application in investment decision based on the data collected from male employees working in private and government sectors in Bhubaneswar city, Odisha,India.

In 2017 Borah-Hazarika [45] discussed some properties of similarity of soft sets. After that they have introduced some new notions such as exact fuzzy soft points, point wise partial similarity and their properties. Also they discussed equality of soft sets based on similarity function. Finally they have shown two applications, one for similarity measure of two face sized and another equality of same composition drugs. WangZhan-Borzooei [96] studied a kind of soft rough semigroups according to Shabir's idea and defined the upper and lower approximations of a subset of a semigroup. According to Zhan's idea over hemirings, they also defined a kind of new $C$-soft sets and $C C$-soft sets over semigroups. In view of this theory, they investigated the soft rough ideals (prime ideals, bi-ideals, interior ideals, quasi-ideals, regular semigroups), finally, they gave two decision making methods: one is for looking a best parameter which is to the nearest semigroup, the other is to choose a parameter which keeps the maximum regularity of regular semigroups.Inthumathi- Chitra- Jayasree [97] provided a soft decision making model on the soft set theory and applied it to real life problem. Based on their method, they analysed a real life problem with different operators and observed that choice of operator is important to get the needful decision. Hence, they concluded that the use of operators on soft sets play an important role in decision making which is flexible. Arafa-Nasef-Kamel [98] applied the theory of soft set to solve a decision making problem in terms of rough mathematics. Ahmad -Hassan [99] defined Einstein product and Einstein sum of vague soft sets and using these operations, They introduced an adjustable approach to vague soft set based decision-making, for solving decisionmaking in uncertain situation. The feasibility of proposed vague soft set based decisionmaking procedure in practical application is shown by some numerical examples.Distance And Similarity Measures For Dual Hesitant Fuzzy Soft Sets And Their Applications In Multicriteria Decision Making Problem is proposed by GargArora [100] in 2017.The dual hesitant fuzzy set is one of the successful extensions of the fuzzy set in which elements are represented in terms of a set of possible values instead of a single number. However, their theory is restricted to their parameterized tool and hence it cannot be effectively applied to a real-life problem. In order to handle it, dual hesitant fuzzy soft set theory has been utilized in this manuscript and hence, based on it, some axioms of distance and similarity measures based on Hamming, Euclidean, and Hausdorff metrics have been proposed here. Various desirable relations between them have also been presented. The proposed distance measures are applied to the field of decision-making under dual hesitant fuzzy soft set environment. Finally, practical examples of pattern recognition and medical diagnoses are given to demonstrate the effectiveness of the proposed measures. A comparative study as well as advantages of the proposed distance measures over existing measures have been presented. Suresh-Ashokkumar [101] investigated the development of theory of soft complex fuzzy set. Consequently, a major part of this work is dedicated to a discussion of the intuitive interpretation of aggregation operation in soft complex fuzzy set. They 
gave an example of possible applications, which demonstrates the applications of aggregation operations that the method can be successfully applied to many problems that contains uncertainties and periodicities. Sonb-Delic-Nguyen [102] proposed the notation of bipolar neutrosophic soft sets that combines soft sets and bipolar neutrosophic sets. Some algebraic operations of the bipolar neutrosophic set such as the complement, union, intersection are examined and they proposed an aggregation bipolar neutrosophic soft operator of a bipolar neutrosophic soft set and developed a decision making algorithm based on bipolar neutrosophic soft sets. Numerical examples are given to show the feasibility and effectiveness of the developed approach.

In 2018 Meryline-Felbin [103] presented a new real measure on the soft real sets and it has shown that any arbitrary soft real set can be embedded in a soft $\mathrm{G}_{\delta}$ set. An application of this measure in solving multi attribute decision making (MADM) problem is illustrated. Fatimah-Rosadi [104 ] motivated and introduced the concept of $\mathrm{N}$-soft set as an extended soft set model. Some useful algebraic definitions and properties are given. They cited real examples that prove that $\mathrm{N}$-soft sets are a cogent model for binary and non-binary evaluations in numerous kinds of decision making problems. Finally, They proposed decision making procedures for $\mathrm{N}$-soft sets.Xie-Jiali [105] investigated limits of interval type of soft sets (for short, $i t$-soft sets). The concept of $i t$-soft sets is first introduced. Then, limits of $i t$-soft sets are proposed and their properties are obtained. Next, point-wise continuity of $i t$-soft sets and continuous $i t$-soft sets are discussed. Finally, an application for rough sets is given. Khalil-Hassan [106] introduced a new soft set called an inverse fuzzy soft set, along with its properties, characteristics, and operations. Then they constructed an algorithm using max-min and min-max decision of inverse fuzzy soft set for a fuzzy decision-making problem. Finally, they applied the algorithm to two decision-making problems to illustrate its applicability. It is shown that proposed approach is viable and provides decision makers a more mathematical insight before making decisions on their options

In 2019 Malathy- Meenakshi [107] discussed the notions of fuzzy soft bi-partite graph, Size and degree of fuzzy soft bi-partite graph and investigated the application of Fuzzy Soft Bi-partite graph in Employee selection for an Organisation.

Data sets produced by hyphenated chemical analysis methods like GC/MS, LC/MS, or HPLC with UV/Vis diode array detection are often presumed to have "trilinear" structure if the profile for each compound does not change shape or position from one sample to the other. By applying this information as a trilinearity constraint in Self Modeling Curve Resolution (SMCR) methods, overlapping peaks related to the pure compounds of interest can be resolved in a unique way. In practice, many systems have non-trilinear behavior due to deviations from ideal response, for example a sample matrix effect, or changes in instrumental response. In such cases, the unique profiles obtained by strictly enforced trilinearity constraints will not necessarily produce true profiles because the data set does not follow the assumed trilinear behaviour.In 2019 Elnaz -Hamid-Paul [108] introduced "soft-trilinearity constraints" to permit peak profiles of given components to have small deviations in their shape and position in 
different samples. They illustrated the influence of soft-trilinearity constraints on the accuracy of SMCR results for the case of a 3-component simulated system and an experimental data set. The results show that implementing soft-trilinearity constraints reduces the range of possible solutions considerably compared to the application of constraints such as just non-negativity. In addition, they show that the application of hard-trilinearity constraints can lead to solutions that are completely wrong or exclude the opportunity of a possible solution at all.The research of incomplete fuzzy soft sets is of paramount importance in fuzzy soft sets, where the combination of incomplete fuzzy soft set and decision-making problem is of great significance. Incomplete information in fuzzy soft sets leads to more uncertainty and ambiguity in decisionmaking.

Wang-Qin [109] proposed an algorithm of fuzzy soft set based decision-making problems under incomplete information. On the basis of the weighted function, they introduced the notions of weighted incomplete soft sets and weighted incomplete fuzzy soft sets, and shown an approach to weighted incomplete fuzzy soft sets for dealing with decision-making. Considering the missing weight function, the concept of incomplete weighted fuzzy soft sets is presented. Meanwhile, they applied the incomplete weighted fuzzy soft sets to solve the decision-making problem. As modalstyle operators for fuzzy soft sets have a precise description of attributes possessed by objects, they applied modal-style operator for incomplete fuzzy soft set to deal with decision-making and proposed a new algorithm to make it more accurate and simple. Hesitant fuzzy set theory is a renowned approach to the formal modelling of uncertain data. An evidence of its success is that it has been extensively used in multi-attribute decision-making problems. Hesitant fuzzy computations make the decision-makers' assessments more flexible and rich, thus improving reliability of the decisions that depend on them. Akram-Arooja-Carlos[110] introduced a novel hybrid model called hesitant fuzzy N-soft sets, which further enhances the virtues of hesitant fuzzy set theory with the benefits of $\mathrm{N}$-soft sets. This theoretical model is capable of incorporating information about the occurrence of ratings or grades in a hesitant environment. They investigated some useful properties of hesitant fuzzy N-soft sets and constructed fundamental operations on them. They developed novel approaches to decision-making including TOPSIS (Technique for Order Preference by Similarity to an Ideal Solution), choice value and L-choice value based on hesitant fuzzy N-soft sets. Finally, they described potential applications of their model and presented the proposed methods as algorithms.

\section{CONCLUSION}

In this paper we have presented a detailed theoretical study on applications of soft sets in decision making problems. Hence we can expect that some research teams will be actively working on soft set applications in the areas of Agriculture, Medicine, social science, soft computing, Artificial Intelligence and data science. 


\section{ACKNOWLEDGMENTS}

The author expresses his gratitude and indebtedness to Prof.N.V.E.S.Murthy and Prof.P.D.N.Srinivasu for their immeasurable support and abundant advice in completing this paper.

\section{REFERENCES}

[1] Zadeh.L.A,Fuzzy sets, Inf. Control, Vol.8, pp.338-353,1965.

[2] Pawlak.Z,Rough sets, Int. J. Comput. Inf. Sci, 11, pp.341-356,1982.

[3] Molodtsov.D,Soft set theory-first result, Comput. Math. Appl, 37, pp.1931,1999 .

[4] Maji.P.K,Roy.A.R ,An Application of Soft Sets in A Decision Making Problem,Computers and Mathematics with Applications 44 ,pp.1077$1083,2002$.

[5] Xiao.Z.L, Chen. B, Zhong and Ye.S, Recognition for soft information based on the theory of soft sets, In: J. Chen, eds., Proceedings of ICSSSM-05, 2, pp. 1104-1106,2005.

[6] Kharal.A and Ahmad.B, Mappings on Fuzzy Soft Classes, Hindawi Publishing Corporation, Advances in Fuzzy Systems Vol 2009, Article ID 407890, 6pages, 2009.

[7] Cagman.N and Enginogh.S, Soft set theory and uni-int Decision making, European journal of operational research 207,2,pp.848-855,2010.

[8] Aktas.H, Cagman.N, Soft sets and soft groups, Information Sciences, Vol.177, pp.2726-2735, 2007.

[9] Ali. M.I, Feng. F, Liu. X, Min. W.K and Shabir. M, On some new operations in soft set theory, Comput. Math. Appl. 57, pp 1547-1553, 2009.

[10] Cagman. N and Enginoglu. S, Soft matrix theory and its decision making, An International Journal of Computers and Mathematics with Applications, Vol59, pp.3308-3314, 2010.

[11] Chen.D, Tsang.E.C.C, Yeung.D.S and Wang.X, Some notes on the parameterization reduction of soft sets, Proceeding of International Conference on Machine Learning and Cybernetics, 3, IEEE Press, pp 1442-1445, 2003.

[12] Kong. Z, Gao.L, Wang.L and Li.S, The normal parameter reduction of soft sets and its algorithm,Computers and Mathematics with Applications 56, pp 3029-3037, 2012.

[13] Xiao.Z, Chen.L, Zhong.B and Shijie. Y, Recognition for soft Information Based on the Theory of Soft Sets, IEEE, pp:1-5, 2005.

[14] Zou. Y and Xiao. Z, Data analysis approaches of soft sets under incomplete 
Information, Knowledge based systems, Vol-21, pp. 941-945,2008

[15] D. Pei and D. Miao, From soft sets to information systems.

[16] A. K M. M. Mushrif, S. Sengupta and. Ray, Texture classification using a novel, soft-set theory based classification, Algorithm, Lecture Notes in Computer Science, 3851, pp. 246-254, 2006.

[17] D. V. Kovkov, V. M. Kolbanov and D. A. Molodtsov, Soft sets theory-based optimization, J. Comput. Sys. Sc. Int., vol. 46(6), pp. 872-880, 2007.

[18] P. Majumdar and S. K. Samanta,Similarity measure of soft sets, New. Math. Nat. Comput., vol. 4(1), pp. 1-12, 2008.

[19] P. K. Maji, R. Biswas and A. R. Roy, Fuzzy soft sets, J. Fuzzy Math., vol. 9(3), pp. 589-602, 2001.

[20] R.K. Bhardwaj, S.K. Tiwari and Kailash Chandra Nayak, A Study of Solving Decision Making Problem using soft set, IJLTEMAS, Vol. IV, Issue IX, pp. 26-32, 2015 .

[21] Z. Xiao, Y. Li, B. Zhong and X. Yang, Research on synthetically evaluating method for business competitive capacity based on soft set, Stat. Methods. Med. Res., pp. 52-54, 2003.

[22] Crossingham B, Marwala T, Lagazio M. Optimised rough sets for modelling interstate conflict. Systems, Man and Cybernetics, SMC 2008. IEEE International Conference on. pp. 1198-1204. IEEE ,2008.

[23] Jiang Y, Tang Y, Chen Q, Wang J, Tang S,Extending soft sets with description logics. Computers \& Mathematics with Applications. 59, pp. 2087$2096,2010$.

[24] S. Das and S. Kar,Group decision making in medical system: an intuitionistic fuzzy soft set approach, Applied soft computing 24 pp.196-211,2014.

[25] Garg.H, Agarwal.N, Tripathi.A,Fuzzy number Intuitionistic fuzzy soft sets and its properties , Journal of Fuzzy Set Valued Analysis ,pp- 196-213, 2016.

[26] Feng.F, Jun.Y.B, Liu.X, Li.L. An adjustable approach to fuzzy soft set based decision making. Journal of Computational and Applied Mathematics. 234, pp.10-20,2010.

[27] Feng.Q, Zhou.Y,Soft discernibility matrix and its applications in decision making, Applied Soft Computing. 24, pp.749-756,2014.

[28] Deli.I, Broumi.S,Neutrosophic soft matrices and NSM-decision making, Journal of Intelligent and Fuzzy Systems.

[29] Roy.A.R and Maji.P.K,A fuzzy soft set theoretic approach to decision making problems, J. Comput. Appl. Math., vol. 203, pp. 412-418, 2007.

[30] Alcantud JCR, A novel algorithm for fuzzy soft set based decision making from multiobserver input parameter data set. Information Fusion ,29, pp.142-148, 
2016.

[31] Majumdar.P, Samanta.S.K,Generalised fuzzy soft sets. Computers \& Mathematics with Applications. 59, pp.1425-1432,2010.

[32] Xiao.Z, Yang.X, Niu.Q, Dong.Y, Gong.K, Xia.S, A new evaluation method based on $D-S$ generalized fuzzy soft sets and its application in medical diagnosis problem, Applied Mathematical Modelling. 36, pp.4592-4604,2012.

[33] Deng.T, Wang .X,An object-parameter approach to predicting unknown data in incomplete fuzzy soft sets, Applied Mathematical Modelling. 37, pp.41394146,2013

[34] Çagman.N, Deli.I,Means of FP-soft sets and their applications, Hacettepe Journal of Mathematics and Statistics. 41, 2012.

[35] Çagman.N, Deli.I,Products of FP-soft sets and their applications, Hacettepe Journal of Mathematics and Statistics. 41,2012.

[36] Deli.I,Çagman.N,Intuitionistic fuzzy parameterized soft set theory and its decision making, Applied Soft Computing. 28, pp.109-113,2015.

[37] Deli.I, Çagman.N,Fuzzy soft games. Filomat. 29, pp.1901-1917,2015.

[38] Gong.K,Wang.P,Xiao.Z,Bijective soft set decision system based parameters reduction under fuzzy environments, Applied Mathematical Modelling. 37, pp.4474-4485,2013.

[39] Wang .C, Qu .A,Entropy, similarity measure and distance measure of vague soft sets and their relations, Information Sciences,pp. 92-106,2013.

[40] Rosenfeld.A, Zadeh.L.A, Fu.K.S, Tanaka.K, Shimuraed.S.M , Fuzzy Sets and Their Applications to cognitive and decision Process,pp. 75 - 95,1975.

[41] Akram.M,Nawaz.S ,Operations on soft graphs, Fuzzy Information and Engineering 7(4),pp. $423-449,2015$.

[42] Mao.J, Yao.D, Wang.C, Group decision making method based on intuitionistic fuzzy soft matrices, Applied Mathematical Modeling 37,pp. 6425-6436,2013.

[43] Yang.Y, Chenli.J,,Fuzzy soft matrices and their applications part I, Lecture notes in Computer Science, pp. 618-627,2011.

[44] Borah.M.J, Neog.T.J and Sut.D.K ,Fuzzy soft matrix theory and its decision making, International Journal of Modern Engineering Research,pp. 121127,2012 .

[45] Rajarajeswari.P and Dhanalakshmi.P,Intuitionistic fuzzy soft matrix theory and its application in decision making, International Journal of Engineering Research and Technology, pp.1100-1111,2013.

[47] Kong. Z, Gao.L, Wang.L, and Li.S, The normal parameter reduction of soft setsand its algorithm.Computers and Mathematics with Applications 56, 30293037, 2008. 
[48] Maji.P.K, Biswas.R, Roy. A.R, Soft set theory, Comput. Math. Appl. 45 555562,2003 .

[49] Zou. Y, and Xiao. Z, Data analysis approaches of soft sets under in complete information, Knowledge Based Systems, 21, 941-945, 2008.

[50] Herawan, T. and Mustafa M.D, A direct proof of every rough set is a soft set. Proceeding of InternationalConference AMS, IEEE Press, 119-124, 2009.

[51] Herawan.T, Rozaida.G, Deris.M.M, Soft Set Theoretic Approach for Dimensionality Reduction, International Journal of Database Theory and Application Vol. 3, No. 2, 2010.

[52] Neog.T.J, Sut.D.K, Application Of Fuzzy Soft Sets In Decision Making Problems Using Fuzzy Soft Matrices,IJMA,Pp-2258-2263,2011.

[53] Cagman.N, Enginoglu.S and Itak. F. C,Fuzzy Soft Set Theory And Its Applications, Iranian Journal of Fuzzy Systems ,Vol. 8, pp. 137-147 ,137,2011.

[54] Razak.S.D and Mohamad.D, A Soft Set based Group Decision Making Method with CriteriaWeight, World Academy of Science, Engineering and Technology, $58,2011$.

[55] Saaty.T.L and Vargus.L.G,Model,Methods,Concepts and Applications of the Analytic Hierarchy Process,USA,Kluwer Academic Publishers,pp.27-43,2001.

[56] Chetia.B and Das.P.K, Application of Vague Soft Sets in students' evaluation. Advances in Applied ScienceResearch, 2 (6),pp 418-423, 2011.

[57] Xun.G and Songlin.Y, Investigations on some operations of soft sets, World Academy of Science,Engineering and Technology,75,2011.

[58] Majumder.P and Samantha. S.K,On Similarity Measure of Fuzzy Soft Sets, Int.J.Advance Soft Comput.Appl,vol.3,No.2, 2011.

[59] Ahmad.N.M, RoseHassan.H, Deris.M.M, Comparison of Techniques in Solving Incomplete Datasets in Softset, International Journal of Database Theory and Application Vol. 4, No. 3, 2011.

[60] Rajpoot.V, Shailendra.k, Shrivastava, Abhishek.M, An Efficient Constraint Based Soft Set Approach for Association Rule Mining, International Journal of Engineering Research and Applications (IJERA) ,2012.

[61] Satya Ranjan.D and Satchidananda.D, A Conspectus of Soft Data Mining in Bioinformatics, CSI Communications, 2012.

[62] Gorzalzany.M .B , A method of inference in approximate reasoning based on interval-valued fuzzy sets, Fuzzy Sets Systems, 21, pp.1-17,1987.

[63] Rajarajeswari.P, Dhanalakshmi.P, An Application of Similarity Measure of Fuzzy Soft Set Based on Distance, IOSR Journal of Mathematics, Vol- 4, PP 27-30, 2012.

[64] Bashir.M, Razak.A.S and Alkhazaleh.S, Possibility Intuitionistic Fuzzy Soft 
Set, Hindawi Publishing Corporation Advances in Decision Sciences, Vol 2012, Article ID 404325, 24 pages, 2012.

[65] Das. P. K , Borgohain.R ,An Application of Fuzzy Soft Set in Multicriteria Decision Making Problem, International Journal of Computer Applications (0975 - 8887), Vol- 38, No.12, 2012.

[66] Xu .W ,Ma.J,Wang.S,Hao.G, Vague soft sets and their properties, Computers \& Mathematics with Applications, , 59(2), pp.787-794, 2010.

[67] Alhazaymeh.K and Hassan.N, Vague Soft Expert Set and its Application in Decision Making, Malaysian Journal of Mathematical Sciences 11(1),pp.23 $39,2017$.

[68] Lashari.S.A, Ibrahim.R and Senan.N, Soft Set Theory for Automatic Classification of Traditional Pakistani Musical Instruments Sounds, International Conference on Computer \& Information Science (ICCIS), 2012.

[69] Jothi.G and Inbarani.H, Soft Set Based Quick Reduct Approach for Unsupervised Feature Selection, IEEE International Conference on Advanced Communication Control and Computing Technologies(ICACCCT),2012.

[70] Blake. C. L and Merz. C. J, UCI Repository of machine learning databases, [Online]. Available:http://www.ics.uci. edul-mlearnl.

[71] Udhaya kumar.U, Inbarani.H.H and Senthil kumar.S, Bijective Soft set based Classification of Medical Data, Proceedings of the International Conference on Pattern Recognition, Informatics and Mobile Engineering (PRIME) ,21-22, 2013.

[72] Saziye .Y, Tugbahan.D, Gulnur.Y, Unal.S, Application of soft sets to diagnose the prostate cancer risk, Journal of Inequalities and Application,Vol 2013,Pp.1-229,2013.

[73] Shabir.M And Naz.M, On Bipolar Soft Sets, arXiv:1303.1344v1,2013.

[74] Karaaslan.F and Karataş.S, $A$ new approach to bipolar soft sets and its applications, Discrete Mathematics, Algorithms and Applications Vol- 07, NO. 04,2015

[75] Majumdar.P, Some Hybrid Soft Sets and Their Application in Decision Making, Applications for Soft Computing, Pages: 32,DOI: 10.4018/978-14666-9798,2016.

[76] Hakim.R.B.F, Sari.E.N, and Herawan.T,Soft Solution of Soft Set Theory for Recommendation in Decision Making, Recent Advances on Soft Computing and Data Mining SCDM , Pp-313-319,2014.

[77] Gogoi.K, Alok .K, Dutta and Chandra .Ch, Application of Fuzzy Soft Set in day to day Problems, International J. of Computer Applications, Vol. 85, No. 7, pp. 27-31, 2014.

[78] Wang.F, Li.X, and Chen.X, Hesitant Fuzzy Soft Set and Its Applications in 
Multicriteria Decision Making,Journal of Applied Mathematics Vol- 2014, Article ID 643785, 10 pages, 2014.

[79] Karaaslan.F, Neutrosophic soft sets with applications in decision making, arXiv:1405 .7964 v2 [cs.AI] , 2014.

[80] Zhang.Xi, On Interval Soft Sets with Applications, Published in Int. J. Comput. Intell. Syst. 2014.

[81] Deli.I ,npn-soft sets theory and their applications, Annals of Fuzzy Mathematics and Informatics, Vol-10, pp. 1-16,2015.

[82] kuei-hu Chang, A more general reliability allocation method using the hesitant fuzzy linguistic term set and minimal variance OWGA weights, Applied Soft Computing,Vol-56, Pp. 589-596,2017.

[83] Congliang.W.U, Chunge.L.I, xiaolan.D, The decision method of basic fuzzy soft set in the application of the asphalt pavement maintenance sorting, Int. Journal of Engineering Research and Applications, Vol. 5, Issue 2, ( Part -1), pp.92-95, 2015.

[84] Alkhazaleh.S,The Multi-Interval-Valued Fuzzy Soft Set with Application in Decision Making,Applied Mathematics, 2015, 6, 1250-1262,Published Online July 2015.

[85] Deli.I, Cagman.N,Application of soft sets in decision making based on game theory, Annals of Fuzzy Mathematics and Informatics Vol-10, pp.1-14,2015.

[86] Sai. B.V.S.T,Nagaraju.D \& Sarma.J.N, A Novel Framework for Dealing with Uncertainty Problems: A Soft Set Based Approach, International Journal of Advanced Research in Computer Science and Software Engineering, ISSN:2277 128X Vol 5, Issue 10, pp.841-848,2015.

[87] Nase, Nashar. E. A,soft set theory and its application in decision making for textiles and apparel marketing,ARTTE Vol. 3, No. 1, ISSN 1314-8788 (print), ISSN 1314-8796 (online), doi: 10.15547/artte.2015.01.004,2015 .

[88] Dauda. M. K, Mamata.M, Waziri.M.Y, An Application Of Soft Set In Decision Making, Jurnal Teknologi (Sciences \& Engineering) 77:13 119122,2015 .

[89] Zakri.A.H, Hossen. H.M, Erwi. L.Y and Sharif. E.A, Application of Soft Sets to Diagnose the Educational Obstacles for Students, Journal of Innovative Technology and Education, Vol. 3, no. 1, pp.61 - 70, 2016.

[90] Cigdem.G and Hande.P, On Some New Operations In Probabilistic Soft Set Theory, European Journal Of Pure And Applied Mathematics, Vol. 9, pp-333$339,2016$.

[91] Hussain.Z.U, IrfanAli.M and Khan.M.Y, Application of Soft Sets to Determine Hamilton Cycles in a Graph, Gen. Math. Notes, Vol. 32, pp.65-79, 2016. 
[92] Mukherjee.A, Sadhan. Sand Debnath.S,Application of intuitionistic Fuzzy soft sets in decision making based on real life problems, CMMA 1, No. 3, pp.1621,2016 .

[93] Rajesh Kumar Pal,Application of Fuzzy Soft Set in Selection Decision Making Problem, International Journal of Engineering Trends and Technology, Vol-42, pp 370-374, 2016.

[94] Sutoyo.E, Mungad.M, Hamid.S, and Herawan.T,An Efficient Soft Set-Based Approach for Conflict Analysis, PLoS One. 2016; 11(2): e0148837,2016.

[95] Nayak. K.C, Rath. B.S\& Mohapatra. S.C, A Soft Set Approach in Investment Decision,IJLTEMAS, ISSN 2278 - 2540 , Vol V, Issue II, 2016.

[96] Qiumei.W, Zhan.J and Borzooei. R.A , A study on soft rough semigroups and corresponding decision making applications, Open Math. 15: pp.1400-1413, 2017.

[97] Inthumathi.V, Chitra.V, Jayasree.S ,The Role of Operators on Soft Sets in Decision Making Problems, International Journal of Computational and Applied Mathematics.Vol-12, pp. 899-910, 2017.

[98] Arafa A. N , Kamel EL and Sayed.M, Molodtsov's Soft Set Theory and its Applications in Decision Making, International Journal of Engineering Science Invention Vol- 6, PP. 86-90, 2017.

[99] Khaleed Ahmad.A, Hassan.N,Einstein-operations on vague soft set, The 4th International Conference On Mathematical Sciences: Mathematical Sciences: Championing the Way in a Problem Based and Data Driven Society, AIP Conference Proceedings 1830(1):070001 , 2017.

[100] Garg.H, Arora.R,Robust aggregation operators for multi-criteria decisionmaking with intuitionistic fuzzy soft set environment, Scientia Iranica ,25(2),pp.931 - 942, 2018.

[101] Thirunavukarasu.P, Suresh.R,Ashokkumar.V, Theory of Complex Fuzzy Soft Set and its Applications, IJIRST -International Journal for Innovative Research in Science \& Technology, Vol. 3 , pp.2349-6010,2017.

[102] Alia.M, Hoang.Le, Sonb, , Irfan.D and Nguyen.D.T, Bipolar neutrosophic soft sets and applications in decision making, Journal of Intelligent \& Fuzzy Systems Vol.33 ,pp.4077-4087,2017.

[103] Meryline.S and Felbin C. K, A study of measure on Soft Real Sets ,International Journal of Pure and Applied Mathematics, Vol. 120 No. 7,pp. 295-303, ISSN: 1314-3395, 2018.

[104] Fatimah.F ,Rosadi.D, Fajriya.R.B, Hakim , José Carlos R. and Alcantud, $\mathrm{N}$-soft sets and their decision making algorithms, Springer, pp.38293842,2018 .

[105] Xie.X and Jiali.H , Limits of it-Soft Sets and Their Applications for Rough Sets, 
Symmetry, 10, 406; doi:10.3390/sym10090406, 2018.

[106] Khalil.A.M and Hassan.N, Inverse fuzzy soft set and its application in decision making, IJIDS, 11(1), pp.73-92, 2019.

[107] Malathy.K and Meenakshi.S, Fuzzy soft bi-partite graph and its Application in Employee Selection for An organisation, International Journal of Recent Technology and Engineering (IJRTE), Vol-7, Issue-5C, 2019.

[108] EInaz.T, Hamid.A and Paul.J.G ,Soft-trilinear constraints for improved quantitation in Multivariate Curve Resolution, The Royal Society of Chemistry 2019.

[109] Wang. Lu and Qin.K ,Incomplete Fuzzy Soft Sets and Their Application to Decision-Making, Symmetry, 11, 535, 2019.

[110] Akram.M,Arooja.A andCarlos.J , Hesitant fuzzy N-soft sets: A new model with applications in decision-making, Journal of Intelligent and Fuzzy Systems, 2019. 